\title{
UM TOQUE NA MASCULINIDADE: a prevenção do câncer de próstata em gaúchos tradicionalistas
}

\author{
Luccas M elo de SOUZA ${ }^{a}$, M ichelli Porto SI LVA ${ }^{b}$, Ingrid de Souza PI N HEIROc
}

\section{RESUM 0}

E studo transversal, com abordagem quantitativa, realizado com 88 gaúchos tradicionalistas participantes do Acampamento Farroupilha de 2009. Objetivou-se verificar a adesão desses gaúchos tradicionalistas aos exames preventivos de câncer de próstata, anal isando, também, quais variáveis influenciam na adesão ao exame de toque retal. Os entrevistados possuíam média de idade de 58,5 anos, com al to salário e el evada escolaridade. G rande parte (92\%) residiu maior tempo em zona urbana, fazia acompanhamento de saúde em instituição particular (70,5\%) e realizou al gum exame preventivo para o câncer de próstata (83\%). Os que fizeram exame preventivo possuíam maior escolaridade, renda e consultavam em instituição particular. Houve menor procura aos exames preventivos por aqueles que moraram a maior parte da sua vida em zona rural. Contudo, não houve relação do local onde residiu o maior tempo com a adesão ao exame de toque retal. Os achados indicam semel hança na adesão aos exames preventivos a outros estudos realizados no Brasil.

D escritores: Enfermagem oncológica. N eoplasias da próstata. E ducação em saúde. M asculinidade.

\section{RESUMEN}

E ste estudio transversal y cuantitativo fue realizado con 88 gauchos tradicionalistas que participaron del Campamento F arroupil ha en 2009. Severificó la adhesión delos participantes a los exámenes preventivos del cáncer depróstata, analizando, también, cuáles variables influyen en la adhesión al examen de tacto rectal. L os encuestados tenían edad media de 58,5 años, con altos salarios y nivel de escolaridad. Gran parte $(92 \%)$ había residido mayor tiempo en las zonas urbanas, hacía acompañamiento de salud en instituciones privadas (70,5\%) ehizo al gún examen preventivo de cáncer depróstata (83\%). L os que habían realizado el examen preventivo tenían mayor nivel de escolaridad, renta y atención de salud en institución privada. H ubo menos demanda de exámenes preventivos por los que habían vivido más tiempo en la zona rural. Sin embargo, no hubo relación entre el lugar de residencia y la adhesión al examen de tacto rectal. $L$ os resultados indican similitud en la adhesión a los exámenes preventivos de otros estudios realizados en B rasil.

Descriptores: E nfermería oncológica. N eoplasias de la próstata. E ducación en salud. M asculinidad.

Título: U n toque a la masculinidad: la prevención del cáncer de próstata en gauchos tradicionalistas.

\section{ABST RACT}

T his cross- sectional and quantitative study was car ried out with 88 traditionalist gauchos, who took part in the Farroupil ha Camp in 2009. It verified their adherence to prostate cancer examination, also analyzing which variables influence in the adherence to digital rectal examination. Participants had an average age of 58.5 years, with high incomeand schooling level. M ost (92\%) lived longer in urban areas, had health follow - up at privatehealth services $(70.5 \%)$ and havehad somepreventive examination for prostate cancer ( $83 \%)$. T he ones who had preventiveex amination had higher education, income and access to private health services. Therewas low er demand for preventive exams by those who lived most of their lives in the rural area. $\mathrm{H}$ ow ever, therew as no relationship betw een the placew her epartici pants lived longer and adherenceto di gital rectal examination. $\mathrm{F}$ indings indicate cor respondence in the adherence to preventive examinations with other studies carried out in B razil.

D escriptors: 0 ncol ogic nursing. P rostatic neoplasms. H ealth education. M asculinity.

$T$ itle: A touch on masculinity: prostate cancer prevention in traditionalist $\mathrm{G}$ auchos.

\footnotetext{
a M estre em Enfermagem, D outorando pelo Programa de Pós-G raduação em Enfermagem da U niversidade Federal do Rio G rande do Sul (UFRGS), Porto Alegre, Rio G rande do Sul, Brasil, Professor A djunto da U niversidade L uterana do Brasil (U L BRA ), Campus G ravataí, Rio Grande do Sul, Brasil.

${ }^{\circ}$ Enfer meira graduada pela U L BRA, Campus G ravataí, Rio G rande do Sul, Brasil.

c A cadêmica de Enfermagem da U L BRA, Campus G ravataí, Rio G rande do Sul, Brasil.
} 


\section{INT RODUÇÃO}

N o Brasil, o câncer (CA) constitui a segunda causa de morte por doenças ${ }^{(1)}$. O CA de próstata é 0 segundo mais prevalente entre os homens, representando aproximadamente $10 \%$ dos cânceres do sexo masculino, atrás apenas do CA de pele não melanoma. Constitui a quarta causa de morte por neoplasias, haja vista que, somente no ano de 2008, foi responsável por 11.955 óbitos. A estimativa para 0 ano de 2010 é de cerca de 52.350 novos diagnósticos no Brasil, com taxa bruta de 53,84 casos para cada 100.000 pessoas. No Estado do Rio G rande do Sul (RS), estima-se 4.500 casos para o ano de 2010, com taxa bruta superior à nacional: 80,40 ocorrências para cada 100.000 habitantes ${ }^{(2)}$. A ssim, o CA de próstata é reconhecido como um problema de saúde pública, necessitando de ações para prevenção, diagnóstico e tratamento ${ }^{(3)}$.

Contudo, encontram-se diver sas dificuldades para a sua prevenção, associadas a fatores como: falta de informação à população; crenças sobre 0 câncer e seu prognóstico; preconceito contra o exame preventivo e a carência de rotinas nos serviços para a prevenção do CA de próstata, dentre outros(4). Além disso, apesar da existência do Consenso Brasileiro sobre o CA de próstata ${ }^{(5)}$, verificase dissenso na literatura sobre aspectos como a necessidade de prevenção, o tipo de exame e a idade ideal para a sua realização.

U ma das formas apontadas pela literatura para rastreamento é o exame de toque retal, procedimento de baixo custo, rápido e que per mite avaliar o tamanho, o formato e a consistência da próstata, embora não em sua total abrangência. A pesar das suas facilidades, acirra o imaginário masculino, sendo interpretado como uma afronta à masculinidade, o que pode influenciar na adesão ao exame $^{(6)}$.

Autores salientam que os homens podem apresentar resistência e constrangimento ao exame de toque retal, pois "viola" a sua masculinidade, no que se refere à condição de ser homem ati$v^{(3,6)}$. A resistência surge, então, porque veem 0 toque retal como algo que conspiraria contra a noção de masculino. N esses casos, a masculinidade é usada como estrutura para a formação da identidade, ditando conceitos a serem seguidos para que sejam reconhecidos como "machos" e não serem questionados por aqueles que possuem as mesmas crenças. N esse sentido, ser homem é um exercício contínuo de negação - mais do que de afirmação negando atributos ditos femininos para se aproximar do que acredita ser a imagem ideal de homem, que foi construída ao longo da vida(7).

No RS, a masculinidade - apontada como barreira para a prevenção do CA de próstata ${ }^{(3)}$ - está presente no modo de viver do gaúcho ${ }^{d}$, afirmada pelo tradicionalismo. I sso preocupa, pois o Estado aparece como um dos que possui as maiores incidências de CA de próstata do país(2).

A ssim, perante o fenômeno CA de próstata e as questões de tradicionalismo/masculinidade, com este estudo objetiva-se verificar a adesão de um grupo de gaúchos tradicionalistas aos exames preventivos para CA de próstata, comparando com a literatura nacional; identificar as variáveis demográficas e socioeconômicas associadas à adesão aos exames preventivos e, especificamente, ao exame de toque retal e identificar o conhecimento desses indivíduos sobre a prevenção do CA de próstata. A ssim pretende-se discorrer se 0 tradicionalismo gaúcho tem influência na adesão ao exame de toque retal.

Destaca-se a importância de estudos que abordem a prevenção do CA de próstata e os modelos hegemônicos de masculinidade, visto existirem poucos estudos sobre 0 assunto. Em geral, as publicações tratam da prevenção do CA de próstata fundamentadas em pesquisas básicas, clínicas e epidemiológicas, havendo pouca discussão no campo da saúde coletiva(3).

\section{MÉTODOS}

Estudo transversal, quantitativo, realizado no Parque M aurício Sirotsky Sobrinho (Parque da $\mathrm{H}$ armonia), em Porto A legre, RS, no transcorrer da comemoração da Semana F arroupilha, em setembro de 2009.

A Semana Farroupilha é realizada anualmente no Parque da Harmonia. E $m$ 2009, 0 acampamento contou com 364 piquetes, que se constituem em tendas ou instalações rústicas, como as moradias dos antepassados, sendo a forma utilizada por grupos ligados à tradição gaúcha para preservar a cultura regional.

\footnotetext{
d Gaúcho é a expressão utilizada para caracterizar as pessoas que nascem no Estado do RS, Brasil. T radicionalismo, sob a ótica da tradição gaúcha, é um estado de consciência que busca preservar 0 passado pelo cultuar, vivenciar e preservar o patrimônio sóciocultural.
} 
A amostra foi constituída por 103 indivíduos. Porém, devido aos critérios da pesquisa, quinze foram excluídos: dez com algum problema na próstata ou sistema urinário, que já estariam em acompanhamento por motivo de patologia (viés de confusão); um considerou-se visitante e quatro moravam fora do RS. Assim, compuseram a amostra 88 gaúchos tradicionalistas.

F oram percorridos todos os piquetes do Parque da $\mathrm{H}$ armonia, buscando interessados em participar da pesquisa. Os critérios de inclusão foram: homens tradicionalistas participantes da Semana Farroupilha 2009, com idade igual ou superior a 51 anos, nascidos e residentes no RS. 0 sujeito deveria responder à seguinte pergunta para ser considerado participante da Semana Farroupilha: você se considera participante ou visitante do Acampamento Farroupilha? (1) participante (2) visitante. Como o A campamento Farroupilha e os seus piquetes são vinculados ao $\mathrm{M}$ ovimento T radicionalista Gaúcho, nesse estudo, os seus participantes são considerados tradicionalistas da cultura gaúcha. 0 corte de idade foi baseado na orientação do Consenso Brasileiro para CA de próstata(5): rastreamento oportunístico (case finding) para homens entre 50 e 70 anos.

Os dados foram coletados por meio de um questionário estruturado entregue aos entrevistados após a leitura e a assinatura do T ermo de Consentimento Livree E sclarecido. Os dados foram analisados utilizando-se o software Statistical Package for the Social Sciences (SPSS) 10.01. U tilizou-se estatística descritiva para a caracterização da amostra (frequência, medidas de tendência central e de dispersão) e os testes $t$ de Student (variáveis simétricas), M ann-W hitney (variáveis assimétricas) e Quiquadrado (variáveis categóricas) para a associação entre os grupos. São consider ados significativos os valores com $p$ bicaudal $\leq 0,05$.

F oram respeitados os princípios éticos da $\mathrm{Re}$ solução no 196/ 96 do Consel ho Nacional de Saúde ${ }^{(8)}$. $O$ projeto foi aprovado pelo Comitê de Ética em Pesquisa da U niversidade Luterana do Brasil (protocolo 2009-227H).

\section{RESULTADOS}

A média de idade dos 88 sujeitos foi de 58,5 anos, e a escolaridade equivalente ao atual Ensino $M$ édio. Verificou-se um alto salário, pois metade dos entrevistados recebia, pelo menos, 4,4 salá- rios mínimos (salário mínimo nacional vigente: $\mathrm{R} \$$ 465,00) (T abela 1).

T abela 1 - Características da amostra. Porto Alegre, RS, 2009.

\begin{tabular}{lc}
\hline Variáveis & $\mathbf{n = 8 8}$ \\
\hline Idade* $^{*}$ Anos de estudo* & $58,5 \pm 6,4$ \\
Renda em salários mínimos & $11,2 \pm 5,5$ \\
Local onde morou mais tempo & $4,4(2,1-7)$ \\
$\quad$ Zona urbana & $81(92,0)$ \\
$\quad$ Zona rural & $7(8,0)$ \\
A companhamento de saúde & \\
$\quad$ A nualmente & \\
$\quad$ Só quando adoece & \\
Rede hospitalar particular/ convênio & $66(76,7)$ \\
História familiar de CA de próstata & $20(23,3)$ \\
Realização do exame preventivo & $62(70,5)$ \\
Exame real izado & $16(18,6)$ \\
$\quad$ PSA & $73(83,0)$ \\
$\quad$ Toque retal & $21(28,7)$ \\
$\quad$ Os dois & $10(13,7)$ \\
Idade no primeiro exame* & $42(57,6)$ \\
\hline
\end{tabular}

* média \pm desvio padrão; ${ }^{\dagger}$ mediana (intervalo interquartis); ; frequência absoluta (porcentual).

Legenda: PSA: Antígeno Prostático Específico.

A média de idade deve-se a um dos critérios de inclusão ter sido idade igual ou superior a 51 anos. A variável "anos de estudo" apresentou média superior à nacional, que é de 7,4 anos de estudo para as pessoas com idade igual ou superior a 18 anos ${ }^{(9)}$, demonstrando, assim, alta escolaridade. A renda alta pode ter facilitado a participação desses indivíduos nas comemorações da Semana Farroupilha, o que move a inter pretação de que o acesso a esse tipo de lazer é mais facil itado para aquel es com maior renda. Entre os que fizeram a prevenção, a média de idade no primeiro exame ficou em 51 anos, corroborando com o Consenso Brasileiro( ${ }^{(5)}$.

Dos participantes, $18,6 \%$ relataram história familiar de CA de próstata, o que é preocupante, visto aumentar em três a dez vezes o risco de adquirir a doença(2). Q uanto à busca por atendimento em saúde, 70,5\% utilizavam a rede particular, reflexo da renda elevada. A maioria $(76,7 \%)$ realizava acompanhamento anual, e 23,3\% apenas quando doentes. Sobre isso, pesquisadores afirmam que os homens buscam os serviços de saúde apenas quando sentem dores insuportáveis, ou quando a situação em que se encontram os impossibilita de trabal har ${ }^{(10)}$. A lém disso, procuram menos o servi- 
ço de saúde comparado às mulheres, pois se consideram mais saudáveis e, devido a questões culturais, veem 0 ambiente de saúde como um local feminino, para pessoas frágeis, ferindo, assim, os conceitos de masculinidade ${ }^{(11)}$. Outros autores salientam o horário de funcionamento dos serviços, o tempo de espera, a falta de unidades específicas e a não resolubilidade do problema no mesmo dia como barreiras para a busca por consulta(10).

D os entrevistados, 73 (83\%) realizaram, ao menos uma vez, um dos exames preventivos. Desses, $57,6 \%$ fizeram o exame de toque retal e dosagem do Antígeno Prostático Específico (PSA), 28,7\% apenas o PSA e 13,7\% somente o toque retal, indicando semel hança na adesão aos exames preventivos para CA de próstata entre esses tradicionalistas e a população brasileira em geral. E studo realizado com professores univer sitários de $\mathrm{M}$ inas $\mathrm{Ge}$ rais (com idade de 51 anos ou mais) constatou que $20,7 \%$ nunca fizer am os exames, $62,1 \%$ realizaram os dois exames, $17 \%$ apenas toque retal e 15,5\% somente o PSA ${ }^{(12)}$. E m pesquisa realizada em dez capitais brasileiras, foram entrevistados 1.061 homens com idade entre quarenta e setenta anos, dos quais $32 \%$ realizaram o exame de toque retal e $47 \%$ o PSA ${ }^{(13)}$.

M esmo se assemelhando à literatura nacional, há uma preocupante parcela $(52,2 \%)$ que não realizou os dois exames preventivos. A prevenção é a única forma de diagnosticar precocemente a doença e auxiliar no tratamento, pois ela é assintomática nos estágios iniciais(3). Em estudo realizado em prontuários de pacientes com diagnóstico de CA de próstata atendidos pelo H ospital de Clínicas de Botucatu, $20 \%$ dos casos foram diagnosticados por exames de rotina, não apresentando sintomatologia. Os demais procuraram o serviço com a doença em estágio avançado, apresentando sintoma ${ }^{(14)}$.

Semelhante aos dados da pesquisa encomendada pela Sociedade Brasileira de U rologia (SBU $)^{(13)}$, mais de $50 \%$ dos homens entrevistados consideraram-se conhecedores dos sinais e sintomas da doença, citando, principalmente, os sintomas urinários como indicativos de problema prostáticos (T abela 2). Outros sintomas lembrados foram: crescimento da próstata, impotência, febre, inapetência, edema nos testículos, fatores psicológicos e PSA elevado, alguns dos quais não relacionados. Observa-se, então, que existem equívocos e mitos sobre os sintomas da doença, necessitando de maior es- clarecimento e investimento na divulgação dos sintomas iniciais (polaciúria, urgência, diminuição do jato urinário, nictúria e gotejamento) e tardios (astenia, perda de peso, anorexia e dor óssea) ${ }^{(15)}$.

Tabela 2 - Conhecimento dos indivíduos sobre 0 câncer (CA) de próstata $(n=88)$. Porto Alegre, RS, 2009.

\begin{tabular}{lc}
\hline Variáveis & $\mathbf{n}(\%)$ \\
\hline Orientado sobre CA próstata & $84(95,5)$ \\
Quem orientou & \\
$\quad$ M édico & $55(63,2)$ \\
F amília/ amigo & $38(43,7)$ \\
M ídia & $33(62,1)$ \\
E nfermeira & $3(3,4)$ \\
Principal exame para detecção & \\
PSA & $20(23,3)$ \\
Toque retal & $39(45,3)$ \\
Os dois & $27(31,4)$ \\
Não conhece os sintomas & $41(46,6)$ \\
Principais sintomas relatados & \\
Jato urinário & $21(23,9)$ \\
Polaciúria & $13(14,8)$ \\
Disúria & $9(10,2)$ \\
N ictúria & $6(6,8)$ \\
Hematúria & $4(4,5)$ \\
\hline
\end{tabular}

Legenda: PSA : Antígeno Prostático Específico.

U ma el evada porcentagem $(46,6 \%)$ de indivíduos relatou desconhecer os sinais e sintomas do CA de próstata, apesar de 95,5\% terem recebido orientação sobre a doença. I sso provoca o questionamento sobre quais os motivos para esse desconhecimento. U ma explicação pode ser o modelo hegemônico (biomédico) de atendimento em saúde no país, haja vista que a maioria dos entrevistados consulta por meio do serviço privado. Tal modelo é centrado, especialmente, na cura e no tratamento de doenças, e as ações de promoção da saúde são pouco incentivadas ${ }^{(16)}$.

H ouve dúvida quanto ao principal exame para prevenção do CA de próstata: a maioria $(45,3 \%)$ citou o exame de toque retal, seguido de $31,4 \%$ que consideraram necessários os exames de toque retal e o PSA concomitantemente, e 23,3\% que acreditam ser suficiente apenas o PSA. I sso talvez se dê pela falta de consenso sobre a prevenção da doença. Pesquisadores ${ }^{(3)}$, ao revisarem a liter atura, encontraram polêmica sobre as recomendações entre o público-alvo e o tipo de exame preventivo aconselhado. Há pesquisadores que recomendam o to- 
que retal associado à dosagem de PSA, enquanto outros orientam a ultra-sonografia transretal quando necessário. Outros, ainda, defendem a realização de apenas um dos exames, questionando o toque retal (pela subjetividade) e o PSA (pela inconclusão). Sobre o público-alvo, encontraram as seguintes orientações: (a) mais de 50 anos ou com 40 anos quando histórico familiar de CA prostático; (b) homens com 45 anos ou com 40 anos, no caso de histórico familiar; (c) 40 anos ou com 35, quando há história familiar; (d) todos os homens com 50 anos ou mais; (e) todos os homens a partir dos 45 anos (recomendação da SBU ) (13); (e) todos os homens a partir dos 40 anos; (f) homens brancos a partir dos 45 anos e negros a partir dos 40 anos; (g) não há necessidade de realização de exame preventivo em assintomáticos. D essa forma, essas divergências podem refletir, também, no conhecimento dos profissionais assistenciais e nas orientações fornecidas por esses à população, o que repercute na baixa adesão aos métodos preventivos.

Sobre a fonte das orientações sobre CA de próstata, a maior participação foi do profissional médico (63,2\%), seguido da mídia (62,1\%). Dados nacionais também revelam uma maior participação da mídia televisiva (50\%) e impressa $(28 \%)$ como informantes, seguida dos médicos $(29 \%)^{(13)}$. E $m$ estudo realizado sobre a divulgação do tema câncer na mídia, encontrou-se 124 publicações em jornais de todo o Brasil, entre os anos de 2003 e
2005. Os principais cânceres abordados foram o de mama, 0 de pele e 0 de pulmão, com pouca divulgação do CA de próstata. Quanto à abordagem, 97 reportagens referiam-se à prevenção, mas apenas uma explicava os métodos de prevenção do CA de próstata. Das 97 matérias, 93,4\% não abordaram os principais sintomas de cada tipo de câncer ${ }^{(17)}$.

N esse estudo, o enfermeiro foi responsável por apenas 3,4\% das orientações, o que preocupa: será que os enfermeiros não orientam sobre CA de próstata? Seria por falta de conhecimento científico, falta de oportunidade ou por despreocupação com a temática?

Nesse sentido, convém salientar que as consultas de enfermagem (momento oportuno para a promoção da saúde) ocorrem, principalmente, em nível de saúde pública (rede básica), e a amostra desse estudo buscou, majoritariamente (70,5\%), atendimento na rede particular, em que a prática da consulta de enfermagem ainda não é uma realidade frequente. No universo das instituições particulares ainda impera o modelo biomédico, centrado no médico como detentor do saber e responsável pelas consultas. T alvez por isso a pouca participação da enfermagem nas orientações a esses homens ${ }^{(16)}$.

Ainda, compararam-se os indivíduos que realizaram exame preventivo (qualquer um dos exames pelo menos uma vez) com os que nunca realizaram (T abela 3).

T abela 3 - Caracterização dos indivíduos quanto à real ização dos exames preventivos para câncer (CA) de próstata (PSA e toque retal). Porto Alegre, RS, 2009.

\begin{tabular}{|c|c|c|c|}
\hline Variáveis & $\operatorname{Sim}(n=73)$ & Não $(n=15)$ & $p$ \\
\hline Idade* & $58,3 \pm 6,3$ & $59,6 \pm 7,1$ & $0,544^{\S}$ \\
\hline Escolaridade* & $11,8 \pm 5,5$ & $7,8 \pm 4,7$ & $0,008^{\S}$ \\
\hline Renda em salários mínimos ${ }^{\dagger}$ & $6,4(3,2-12,9)$ & $1,9(1-4,3)$ & $0,001^{\prime \prime}$ \\
\hline L ocal onde morou mais tempo & & & 0,058 \\
\hline Zona urbana & $69(94,5)$ & $12(80,0)$ & \\
\hline Zona rural & $4(5,5)$ & $3(20,0)$ & \\
\hline A companhamento de saúde & & & 0,0119 \\
\hline Instituição particular & $51(70,8)$ & $4(33,3)$ & \\
\hline Instituição pública & $21(29,2)$ & $8(66,7)$ & \\
\hline Sistema de saúde utilizado & & & $0,027 \pi$ \\
\hline SUS & $18(24,7)$ & $8(53,3)$ & \\
\hline Convênio/ particular & $55(75,3)$ & $7(46,7)$ & \\
\hline
\end{tabular}

* média \pm desvio padrão; ${ }^{\dagger}$ mediana (intervalo interquartis); ${ }^{\ddagger}$ frequência absoluta (porcentual); ${ }^{\S}$ t de Student; $"$ M ann-W hitney; " Quiquadrado de Pearson.

Legenda: PSA: Antígeno Prostático E specífico; SU S: Sistema Ú nico de Saúde.

A queles que fizer am pelo menos um dos exames possuíam maior escolaridade e renda, e reali- zavam acompanhamento de saúde em instituição particular por meio de convênio ou consulta parti- 
cular. A diferença estatística foi significativa, indicando que possuir maiores recursos financeiros auxilia na busca por atendimento devido à facilidade ao acesso, e que a escolaridade está associada à maior consciência sobre a saúde.

I dade também é um determinante para a prevenção do CA de próstata, pois homens mais novos referem não ter muitas restrições aos exames ${ }^{(7)}$. Entretanto, no presente estudo, não se verificou esse comportamento.

Os sujeitos foram questionados sobre a cidade em que viveram por mais tempo, a fim de in- vestigar diferença na procura por exames preventivos conforme o local de origem. A hipótese é que homens com raízes rurais cultivem mais as tradições culturais gaúchas, cultuando a masculinidade. 0 fato de ter morado maior tempo em zona rural mostrou tendência para a não realização dos dois exames preventivos $(p=0,058)$. Para esse achado, têm-se duas suposições: em zonas rurais existem maiores dificuldades de acesso aos serviços de saúde, bem como há maior solidez nas questões culturais e tradicionalistas, dificultando a procura por atendimento ( $T$ abela 4).

T abela 4 - Caracterização dos indivíduos quanto à realização ao exame de toque retal. Porto Alegre, RS, 2009.

\begin{tabular}{|c|c|c|c|}
\hline Variáveis & $\operatorname{Sim}(n=52)$ & Não (n = 36) & $\mathrm{p}$ \\
\hline I dade* & $58,5 \pm 6,4$ & $58,6 \pm 6,5$ & $0,917^{\S}$ \\
\hline Escolaridade* & $12,5 \pm 5,5$ & $9,2 \pm 5,1$ & $0,060^{\S}$ \\
\hline Renda em salários mínimos ${ }^{\dagger}$ & $7,7(3,4-15)$ & $2(1,6-5,8)$ & $0,001^{\prime \prime}$ \\
\hline Local onde morou mais tempo ${ }^{\ddagger}$ & & & $0,363^{n}$ \\
\hline Zona urbana & $49(94,2)$ & $32(88,9)$ & \\
\hline Zona rural & $3(5,8)$ & $4(11,1)$ & \\
\hline A companhamento de saúde f $^{\ddagger}$ & & & $0,005^{\natural}$ \\
\hline Instituição particular & $40(76,9)$ & $15(46,9)$ & \\
\hline Instituição pública & $12(23,1)$ & $17(59,1)$ & \\
\hline Sistema de saúde utilizado ${ }^{\ddagger}$ & & & $0,036^{\pi}$ \\
\hline SUS & $11(21,2)$ & $15(41,7)$ & \\
\hline Convênio/ particular & $41(78,8)$ & $21(58,3)$ & \\
\hline
\end{tabular}

* média \pm desvio padrão; ${ }^{\dagger}$ mediana (intervalo interquartis); ${ }^{\ddagger}$ frequência absoluta (porcentual); ${ }^{\S} \mathrm{t}$ de Student; ${ }^{\prime \prime}$ M ann-W hitney; ${ }^{\circledR}$ Q uiquadrado de Pearson.

Legenda: SU S: Sistema Ú nico de Saúde.

Compararam-se os indivíduos que fizeram 0 exame de toque retal com aqueles que não o realizaram. 0 grupo que realizou o exame de toque retal $(n=52)$ possuía maior renda e utilizava o serviço de saúde de instituições particulares via convênio ou consulta particular $(p<0,05)$. Quem possuía maior escolaridade tendeu à realização do exame de toque $(p=0,06)$.

A pesar da maior porcentagem de realização do exame de toque retal naquel es que moraram a maior parte do tempo em zona urbana, não houve diferença significativa entre os grupos. Foi realizado tal questionamento pressupondo-se que tradicionalistas com origens rurais poderiam apresentar maiores barreiras culturais e comportamentais ao exame de toque retal. Assim, partindo desse pressuposto, o tradicionalismo gaúcho não influenciou na adesão ao exame de toque retal, considerando a hipótese do tradicionalismo e das questões de masculinidade serem mais fortes nas zonas rurais. Supõe-se, então, que a escolaridade e a renda el evada sobrepujaram o preconceito ao exame de toque, como indicam outros estu$\operatorname{dos}^{(6,7)}$

Os sujeitos também foram questionados sobre 0 motivo de nunca realizarem o exame de toque retal, e relataram que a não realização do exame deveu-se ao fato do médico nunca ter solicitado $(15,9 \%)$, por considerarem-se saudáveis $(10,2 \%)$, por descuido/ esquecimento (10,2\%), por falta de tempo $(6,8 \%)$, por confiança no exame do PSA $(6,8 \%)$, por preconceito $(5,7 \%)$ e por medo $(3,4 \%)$. $\mathrm{N}$ esse sentido, é fundamental o papel da enfermagem, alicerçado na prática da educação em saúde, despertando a consciência crítica das pessoas e dos grupos sociais, envolvendo-os nos aspectos relacionados à sua saúde e transformando a realidade social|(16). 


\section{CONSIDERAÇÕES FINAIS}

Verificou-se que os tradicionalistas gaúchos possuíam al ta escolaridade e renda, com média de idade de 58,5 anos. A maioria (83\%) realizou, ao menos uma vez, um dos exames preventivos, sendo que $57,6 \%$ fizeram o exame de toque retal e dosagem do PSA, $28,7 \%$ apenas o PSA e $13,7 \%$ apenas o toque retal. G rande parte $(46,6 \%)$ relatou desconhecer os sinais de sintomas do CA de próstata, mesmo que a maioria absoluta tenha recebido orientação sobre a doença.

Como o A campamento Farroupilha é um Iocal de culto à tradição gaúcha e à masculinidade, poderia se considerar que os tradicionalistas pudessem aderir menos aos exames preventivos de próstata em comparação a outros homens, em especial ao exame de toque retal. N o entanto, os resultados assemelham-se aos de outros estudos conduzidos no Brasil. Verificou-se, então, que o tradicionalismo gaúcho parece não influenciar na adesão aos exames preventivos para CA de próstata, pois se encontrou semelhança de adesão aos estudos nacionais. Entretanto, variáveis como renda, escolaridade e acesso aos serviços de saúde estão associadas.

Acredita-se que a menor procura aos exames preventivos naqueles que moraram a maior parte em zona rural esteja associada à dificuldade de acesso ao serviço de saúde, e não às questões de tradicional ismo/masculinidade, haja vista não haver influência do local onde residiu na adesão ao exame de toque retal - o que, nesta perspectiva, vioIaria a masculinidade de forma mais acentuada.

Contudo, pelas limitações de um delineamento transversal, é preciso uma inter pretação cautelosa, pois não se pode chegar a resultados definitivos. D entre as limitações, salienta-se a alta renda e escolaridade dos entrevistados, que pode ter velado o preconceito das questões de masculinidade. Recomenda-se a realização de outras pesquisas investigando a relação da masculinidade com a prevenção do CA de próstata, utilizando populações com outro perfil de escolaridade e renda, a fim de se verificarem congruências/ divergências com este estudo.

Cabe citar ainda a pouca participação da enfermagem com relação às orientações sobre a doença, servindo como alerta. Será que os enfermeiros não estão preocupados com a prevenção do CA de próstata? Como mudar essa realidade?

\section{REFERÊNCIAS}

1 Souza LM , G orini M IPC. Diagnósticos de enfermagem em adultos com leucemia mielóide aguda. Rev G aúcha E nferm. 2006;27(3):417-25.

2 M inistério da Saúde (BR), Instituto N acional do Câncer. E stimativa 2010: incidência de câncer no Brasil. Rio de Janeiro; 2009.

3 G omes R, R ebello LEF S, A raújo FC, N ascimento EF. A prevenção do câncer de próstata: uma revisão de literatura. Ciênc Saúde Colet. 2008;13(1):235-46.

4 M aia KO, M oreira SH, Filipini S M. Conhecimento e dificuldade em rel ação à prevenção do câncer de próstata na ótica dos homens de meia idade. In: A nais do 13o Encontro Latino A mericano de I niciação Científica, 9o E ncontro Latino A mericano de Pós-G raduação: ciência e ética, o paradigma do século XXI; 2009 out 16-17; São José dos Campos, Brasil [ Internet]. São Paulo: U N IVAP; 2009 [ citado 2009 set 10] . Disponível em: http:// www.inicepg.univap.br/cd/ I N IC_2009/ anais/arquivos/0132_0028_01.pdf.

5 M inistério da Saúde (BR), Instituto N acional do Câncer. Câncer de próstata: consenso. Rio deJaneiro; 2002.

6 G omes R, N ascimento EF, Rebello LE FS, A raújo FC. As arranhaduras da masculinidade: uma discussão sobre o toque retal como medida de prevenção do câncer prostático. Ciênc Saúde Colet. 2008;13(6):197584.

7 Nascimento M R. Câncer de próstata e masculinidade: motivações e bar reiras para a realização do diagnóstico precoce da doença [ I nternet] . Caxambu: A besp; 2000 [ citado 2009 set 15]. Disponível em: http:// www.abep.nepo.unicamp.br/ docs/ anais/ pdf/ 2000/ T odos/ C \%C 3\%A 2ncer\%20de\%20P r\%C 3\%B 3stata\% $20 \mathrm{e} \% 20 \mathrm{M}$ asculinidade $\% 20-\% 20 \mathrm{M}$ otiva $\%$ C 3\%A 7 Ses $\% 20 \mathrm{e} \% 20$ Bar reiras...pdf.

8 M inistério da Saúde (BR), Conselho Nacional de Saúde. Resolução 196, de 10 de outubro de 1996: diretrizes e normas regulamentadoras de pesquisa envolvendo seres humanos. Brasília (D F ); 1996.

9 Instituto Brasileiro de G eografia e Estatística. Pesquisa nacional por amostra de domicílios: síntese de indicadores 2008. Rio de Janeiro; 2009.

10 G omes R, Nascimento EF, A raújo F C. Por que os homens buscam menos os serviços de saúde do que as mulheres? As explicações de homens com baixa escolaridade ehomens com ensino superior. Cad Saúde Pública. 2007;23(3):565-74. 
11 Souza L M , M orais EP, Barth QCM . Características demográficas, socioeconômicas e situação de saúde de idosos de um programa de saúde da família de Porto A legre, Brasil. Rev L atino-Am Enfermagem. 2006;14(6):901-6.

12 M iranda PSC, Côrtes M CJW, M artins M E, Chaves PC, Santarosa RC. Práticas de diagnóstico precoce de câncer de próstata entre professores da faculdade de medicina - U F M G. Rev Assoc M ed Bras. 2004;50 (3):272-5.

13 Sociedade Brasileira de U rologia. A penas $32 \%$ dos homens já fizeram exame de toque [ Internet]. Rio de Janeiro; 2009 [ citado 2009 dez 02]. Disponível em: http:/ / www.sbu.org.br/ 2010/ 03/ 17112009apenas-32-dos-homens-ja-fizeram-exame-de-toque/
14 G oncalves I R, Padovani CS, Popim RC. Car acterização epidemiológica e demográfica de homens com câncer de próstata. Ciênc Saúde Colet. 2008;13(4): 1337-42.

15 Rodrigues $A B$. Câncer geniturinário e do aparelho reprodutor masculino. In: M ohallem AGC, Rodigues A B. E nfermagem oncológica. Barueri: M anole; 2007. p. 300-15.

16 Souza L M , W egner W, G orini M IPC. E ducação em saúde: uma estratégia de cuidado ao cuidador leigo. Rev L atino-Am Enfermagem. 2007;15(2):167-74.

17 Jurberg C, G ouveia M E, Belisário C. N a mira do câncer: o papel da mídia brasileira. Rev Bras Cancerol. 2006;52(2):139-46.
Endereço do autor / Dirección del autor / Author's address:

Luccas M elo de Souza

Av. Itacolomi, 3600, São Vicente

94170-240, G ravataí, RS

E-mail:luccasms@gmail.com
Recebido em: 15/ 09/2010

A provado em: 06/ 01/ 2011 